\title{
Developmental dyscalculia and basic numerical capacities: a study of 8-9-year-old students
}

\author{
Karin Landerl ${ }^{\mathrm{a}, \mathrm{b}}$, Anna Bevan ${ }^{\mathrm{a}}$, Brian Butterworth ${ }^{\mathrm{a}, *}$ \\ ${ }^{\mathrm{a}}$ Institute of Cognitive Neuroscience, University College London, 17 Queen Square, London WC1N 3AR, UK \\ ${ }^{\mathrm{b}}$ Department of Psychology, University of Salzburg, Salzburg, Austria
}

Received 21 March 2003; revised 17 September 2003; accepted 13 November 2003

\begin{abstract}
Thirty-one 8- and 9-year-old children selected for dyscalculia, reading difficulties or both, were compared to controls on a range of basic number processing tasks. Children with dyscalculia only had impaired performance on the tasks despite high-average performance on tests of IQ, vocabulary and working memory tasks. Children with reading disability were mildly impaired only on tasks that involved articulation, while children with both disorders showed a pattern of numerical disability similar to that of the dyscalculic group, with no special features consequent on their reading or language deficits. We conclude that dyscalculia is the result of specific disabilities in basic numerical processing, rather than the consequence of deficits in other cognitive abilities.
\end{abstract}

(C) 2004 Published by Elsevier B.V.

Keywords: Dyscalculia; Numerical processing; Intelligence; Working memory; Arithmetic development

\section{Introduction}

Mathematics is a complex subject, involving language, space and quantity. Much research into the development of mathematical skills has focused upon relatively basic numerical abilities, such as arithmetic or counting (Bisanz, 1999a), but even at such early levels many complex abilities are involved. These include transcoding between spoken number words and Arabic numerals, relating these to semantic representations of set size ("numerosity"), reasoning about relative set sizes (if 1 is added to 2 , the result should be 3); and understanding the relations between set size and counting order.

\footnotetext{
* Corresponding author. Fax: +44-71-580-1100.

E-mail address: b.butterworth@ucl.ac.uk (B. Butterworth).
} 
The complexity of numerical processing has made defining what it means to have a specific mathematical learning disability (dyscalculia) difficult. Traditional definitions (e.g. DSM-IV, American Psychiatric Association, 1994) state that the child must substantially underachieve on a standardized test relative to the level expected given age, education and intelligence, and must experience disruption to academic achievement or daily living. Standardized achievement tests, however, generally test a range of skills, which may include spatial and verbal abilities, before collapsing the total into one global score of 'maths achievement'. There is thus a substantial risk of Type I errors. In addition standardized tests are diverse, so what is meant by 'maths achievement' may vary substantially between tests. For this reason it has been hard for researchers to pinpoint the key deficits in dyscalculia, or to be sure how to define dyscalculics for study.

A range of terms for referring to developmental maths disability has emerged, along with different criteria. Geary and colleagues use the term "mathematical disabilities" and include all children who fall below the 30th percentile (Geary, Hoard, \& Hamson, 1999) or 35th percentile (Geary, Hoard, \& Hamson, 2000) on the Woodcock-Johnson Mathematics reasoning test (Woodcock \& Johnson, 2001). Jordan and colleagues (Hanich, Jordan, Kaplan, \& Dick, 2001; Jordan, Hanich, \& Kaplan, 2003a; Jordan, Kaplan, \& Hanich, 2002) refer to "mathematics difficulties", and include all children below the 35th percentile of the Woodcock-Johnson Broad Mathematics Composite Score. The 35 th percentile criterion means that the best children will be about 0.39 SD units below the mean, and that $90 \%$ of the sample will be better than 2 SDs below the mean. Koontz and Berch (1996) use the term "arithmetic learning disabilities" and include children below the 25th percentile on the Iowa Test of Basic Skills. Most children so classified would fall between 0.67 and 1.18 SDs below the expected mean, and could thus be regarded as in the low average or even the average range. These authors' terminology, as well as criteria, make it clear that they are considering a range of causes for low mathematics achievement, not just the clinical condition of dyscalculia.

Several authors refer to "dyscalculia" or "developmental dyscalculia". They tend to use their own tests and a much more stringent criterion. Shalev, Manor, and Gross-Tsur (1997), who have carried out the most extensive study of this condition, use the criterion of two grades below chronological age. Butterworth's (2003) Dyscalculia Screener requires scores on two tests to be in the lowest two stanines (11th percentile). The study reported here used teachers' report and a criterion of 3 SDs below the mean of the control group to avoid false positives. For this group, we will use the term 'dyscalculia' (abbreviated to DC).

\subsection{Features of dyscalculia}

The most generally agreed upon feature of children with dyscalculia is difficulty in learning and remembering arithmetic facts (Geary, 1993; Geary \& Hoard, 2001; Ginsburg, 1997; Jordan, Hanich, \& Kaplan, 2003b; Jordan \& Montani, 1997; Kirby \& Becker, 1988; Russell \& Ginsburg, 1984; Shalev \& Gross-Tsur, 2001). A second feature of children with dyscalculia is difficulty in executing calculation procedures, with immature problemsolving strategies, long solution times and high error rates (Geary, 1993).

Temple (1991) has demonstrated using case studies that fact retrieval and procedural difficulties are dissociable in dyscalculia. However, case studies, while providing 
important theoretical information on cognitive structures, are not necessarily representative of the majority of dyscalculic children: such dissociations may be rare. Ashcraft, Yamashita, and Aram (1992) found no dissociation between arithmetic fact ability and procedural ability in children with numerical processing difficulties. Russell and Ginsburg (1984) found that a group of children with "mathematics difficulties" struggled with both written calculation and arithmetic fact retrieval. Geary (1993) suggests that procedural problems are likely to improve with experience, whereas retrieval difficulties are less likely to do so. Geary proposes that this dissociation emerges because procedural problems are due to lack of conceptual understanding, while retrieval difficulties are the result of general semantic memory dysfunction. However, it is possible that both difficulties result from a lack of conceptual understanding. It may be easier for a child to memorise one or two meaningless procedures than the multitude of arithmetic facts (from simple number bonds to multiplication tables) which, without understanding of cardinality, are simply unrelated word strings.

Researchers, then, agree that dyscalculia manifests itself as a problem in learning arithmetic facts and calculation procedures. The question which remains unanswered relates to the underlying deficits which cause these problems. Various candidates have been put forward, including dyslexic difficulties, memory difficulties, spatial difficulties and attentional difficulties. However, many of the studies which have been designed to relate these "underlying" abilities to dyscalculia have confounded them with numerical processing abilities.

\subsection{Underlying processing deficits}

One approach to the study of developmental dyscalculia involves identifying the cognitive and neuropsychological correlates of dyscalculia in an attempt to extrapolate causal features or to identify dyscalculia subtypes with differential causes. Suggested cognitive causes include abnormal representations in semantic memory and difficulty with working memory.

Geary and colleagues (Geary et al., 2000; Geary \& Hoard, 2001) have suggested that semantic memory difficulties may underlie the problems experienced by developmental dyscalculics in learning number facts, and may also underlie the comorbid reading difficulties frequently found with dyscalculia. The argument is based on evidence that dyscalculic children have difficulty learning and remembering arithmetic facts. However, if this theory is correct, we should expect all dyslexic children to have number fact problems and vice versa. In addition, the argument confounds semantic memory with numerical processing. There is little empirical evidence for a non-numerical semantic deficit in dyscalculic children. To our knowledge only one study has found such evidence: Temple and Sherwood (2002) found that a group of children with arithmetic difficulties were slower at colour and object naming than controls, evidence for a generalized speed of access difficulty in this sample. However, the authors argued against a causal relationship between speed of access and arithmetic ability, one reason for caution being the small size of the group (four participants). Another problem with the hypothesis arises in the light of evidence that semantic memory for numbers is mediated by a different system than general semantic memory. Neuropsychological studies indicate that number knowledge is 
dissociable from verbal semantic memory (Cappelletti, Butterworth, \& Kopelman, 2001), and that the semantic memory systems for numerical and non-numerical information are localized in different areas of the brain (Thioux, Seron, \& Pesenti, 1999). This functional and anatomical dissociation between the two memory systems makes it unlikely that the same semantic deficit can account for both maths and reading disability.

Working memory difficulties have also been associated with developmental dyscalculia. Geary (1993) suggests that poor working memory resources not only lead to difficulty in executing calculation procedures, but may also affect learning of arithmetic facts. In general the aspect of working memory that has been focused on is the phonological loop (Baddeley, Lewis, \& Vallar, 1984), normally assessed by the number of spoken items (generally digits) which can be remembered in the correct sequence. However, empirical evidence for a correlation between reduced span in phonological working memory and developmental dyscalculia is conflicting, and the issue is confused by potential confounds. Reduced phonological span is associated with general academic difficulties and with dyslexia, both of which are also associated with difficulties in mathematics, and, presumably, dyscalculia. Therefore, any study examining Working memory in dyscalculic children should control for both reading ability and general IQ.

Siegel and Ryan (1989) found that children with dyscalculia did less well than controls on a working memory task involving counting and remembering digits, but not on a nonnumerical Working memory task. This led them to speculate that there is a working memory system specialised for numerical information, and that children with dyscalculia have specific problems with this system. Similarly, McLean and Hitch (1999) found a trend towards poorer digit span in dyscalculic children, while there was no difference on a non-numerical task testing phonological working memory (non-word repetition). No evidence was found for a faster decay rate of phonological representations in dyscalculic children. These authors concluded that dyscalculic children do not have reduced phonological working memory capacity in general, although they may have a specific difficulty with working memory for numerical information. On the other hand, they found that spatial working memory and some aspects of central executive function were poorer in dyscalculic children.

Koontz and Berch (1996) tested children with and without dyscalculia using both digit and letter span (the latter being a measure of phonological working memory capacity which is not confounded with numerical processing). This study found that dyscalculic children performed below average on both span tasks, indicating general working memory difficulties. However, although children in this study were above the 30th percentile on a standardized reading achievement test, general IQ was not controlled. In contrast, Geary et al. (1999) did not find a difference between dyscalculic children and controls on the forward digit span measure, but did find a difference on backward digit span, thought to tap central executive processes.

Temple and Sherwood (2002) attempted to resolve this confusion, testing children with dyscalculia and controls on forward and backward digit span, word span and Corsi blocks. This study found no differences between groups on any of the working memory measures, and no correlation between the working memory measures and measures of arithmetic ability. 
On balance, although various forms of working memory difficulty may well co-occur with maths difficulties, there is little agreement between these studies, and no convincing evidence implicating any form of working memory as a causal feature in dyscalculia.

\subsection{Subtyping}

Another approach to the study of developmental dyscalculia has involved subtyping dyscalculics according to the presence or absence of other disorders, in an attempt to highlight underlying processes which may contribute to the comorbidity of the disorders. An important correlate of maths disability is reading disability. It is estimated that $40 \%$ of dyslexics also have maths disability (Lewis, Hitch, \& Walker, 1994). One of the most common ways of subtyping dyscalculic children is according to whether or not they have a comorbid reading disability. Hanich et al. (2001) found that "children with MD-only seem to be superior to children with MD/RD in areas that may be mediated by language but not in ones that rely on numerical magnitudes, visuospatial processing, and automaticity".

Rourke and his colleagues (see Rourke, 1993 for a review) have compared children with arithmetic difficulties only and children with better arithmetic scores than reading scores. Children with arithmetic difficulties only were more likely to have difficulties with spatial and psychomotor abilities, whilst children with reading difficulties were more likely to struggle with verbal tasks. The authors suggest that these findings indicate that comorbid maths and reading difficulties result from left-hemisphere dysfunction, while specific difficulty with maths stems from right-hemisphere dysfunction. However, Rourke's constellation of 'right-hemisphere' symptoms is similar to the 'left-parietal' constellation found in Gerstmann's syndrome (Gerstmann, 1940). In addition, a recent attempt to replicate Rourke's findings (Shalev et al., 1997) failed; Shalev et al. found no qualitative difference between children with both reading and maths disability and children with maths disability only. Children with both disorders scored more poorly on several measures, but the authors concluded that this was unsurprising, given that the presence of more than one disorder indicates relatively widespread brain dysfunction. Fayol, Barrouillet, and Marinthe (1998) attempted to test Rourke's hypothesis regarding the causal relationship between neuropsychological deficits and arithmetic difficulties. They conducted a longitudinal study in which nursery school children were given tests of finger agnosia, graphisthesia and simultagnosia. These neuropsychological measures correlated with simple arithmetic tests given at the same time. However, except (oddly) for word problem solving, general intelligence in nursery school was a better predictor of arithmetic in the 1st year of school than were the neuropsychological tests. This finding suggests that correlation, in this case, is not causation.

Another set of deficits which are associated with developmental dyscalculia are finger agnosia, dysgraphia and difficulties with left-right discrimination. Taken together this symptom complex constitutes developmental Gerstmann's syndrome. However, since it appears that the four symptoms can appear individually and in any combination, and are frequently associated with other conditions such as reading disability (Kinsbourne \& Warrington, 1963; Spellacy \& Peter, 1978) it is unlikely that the symptoms are related in terms of a single underlying deficit. 
Other conditions which have been associated with dyscalculia are ADHD (Badian, 1983; Rosenberg, 1989; Shalev et al., 1997), poor hand-eye co-ordination (Siegel \& Feldman, 1983), poor memory for non-verbal material (Fletcher, 1985), and poor social skills (Rourke, 1989). Shalev and Gross-Tsur (1993) examined a group of seven children with developmental dyscalculia who were not responding to intervention. All seven were suffering numbers, additional neurological conditions, ranging from petit mal seizures through dyslexia for numbers, attention deficit disorder and developmental Gerstmann's syndrome. In summary, while it is clearly the case that dyscalculia is frequently comorbid with other disabilities, causal relationships between the disorders have not been proven. In addition, the utility of subtying dyscalculics according to neuropsychological or cognitive correlates will not be clear until it has been shown that the different subtypes display qualitatively different patterns of numerical deficit.

Relatively few studies have examined differences between subtypes on tasks involving numerical processing. Shalev et al. (1997) found that children with comorbid maths and reading difficulties were more profoundly impaired than children with specific maths problems on subtraction and division and had lower verbal IQ scores. They also scored consistently lower on most of the WISC subtests, although this difference did not reach statistical significance. However, the pattern of numerical impairment was the same for both groups. This study found no evidence for a dissociation between the two groups in numerical processing, although children with comorbid maths and reading difficulties tended to be more impaired than children with specific maths problems.

Jordan and Montani (1997) compared a group of children with specific maths disability with a group of children who had maths disability in the context of more general academic difficulties. Children with maths disability only were better able to execute backup strategies in arithmetic, and were able to perform at a normal level under untimed conditions, although their performance dropped under timed conditions. Children with more general difficulties struggled under both conditions. The authors suggested that children with specific maths difficulties are able to compensate under untimed conditions because of relatively good verbal or conceptual skills. However, although this study also indicates that children with general difficulties have quantitatively more difficulty than children with specific maths disability, again there is no evidence that the pattern of numerical impairment is qualitatively different between the two groups. More detailed examination of the numerical abilities of groups of children are in order before it is certain that subtyping developmental dyscalculics according to this framework is a useful approach.

It is clear that dyscalculia frequently co-occurs with a range of other disabilities. However, it is still far from clear that these disabilities play any causal role in developmental maths disability. Not only has no single underlying process been identified which predicts dyscalculia, but also there is no evidence for qualitatively different patterns of impairment across dyscalculia subtypes, as would be expected if different subtypes corresponded to different underlying causes. There is no robust empirical evidence causally relating any of these correlates to numerical ability. In addition there is very little coherent theory which could explain such causal relationships. Currently the most likely explanations for overlap between different disabilities are anatomical or genetic: damage to a brain area or failure of that area to 
develop normally may affect one or more cognitive functions depending on the extent and severity of the damage (Shalev \& Gross-Tsur, 1993).

\subsection{Dyscalculia as a deficit in a specialized brain system}

The studies described above have attempted to get at the root of dyscalculia by examining various abilities, not obviously related to number processing, which are hypothesized to underlie dyscalculia. This approach involves an implicit assumption that the representation and manipulation of numerical information is a higher-order Rinction which is dependent upon the abilities described. However, evidence from neuropsychology and research with animals and very young children suggests that number processing is not only independent of other abilities, but is also manifested at a very basic level. Numerical abilities, including arithmetic, are mediated by areas in the parietal lobe (Dehaene, Dehaene-Lambertz, \& Cohen, 1998). Neuropsychological evidence has shown that the ability to understand numbers and to calculate is dissociable from language (Cohen, Dehaene, Cochon, Lehericy, \& Naccache, 2000); from semantic memory for nonnumerical information (Cappelletti et al., 2001); and from working memory (Butterworth, Cipolotti, \& Warrington, 1996). There is also evidence for a genetic basis for this specialization in studies of Turner's Syndrome (Butterworth et al., 1999).

Not only are numerical abilities independent of other abilities, but also appear to be 'hardwired' and are manifested even in infants (Starkey, Spelke, \& Gelman, 1990; Wynn, 1995). A number of studies have found numerical processing abilities in animals (see Gallistel, 1990 for a review). Thus number processing appears to be a function which emerges in infants at a very early age, and is independent of other abilities. This argues against a role for language-related abilities such as semantic or working memory in developmental dyscalculia. It seems likely that basic numerical functions, such as comprehension of numerical symbols, counting, and simple calculation, are built primarily upon early mechanisms for processing small numerosities. These mechanisms also seem to be a good candidate for a basic deficit underlying dyscalculia.

If dyscalculia is the result of a fundamental difficulty with numerical processing, as is proposed here, dyscalculic children should have problems with even the most basic functions involving numbers such as subitizing, counting small numbers of objects, using number names and numerals, and comparing numerical magnitudes, as well as more advanced arithmetical skills.

Some evidence for this comes from a study by Koontz and Berch (1996), who found that the dyscalculic children appeared to be counting to 3 rather than subitizing in a dotmatching task. Kirby and Becker (1988) found a trend for dyscalculic children to be slower at number naming relative to controls, while Geary et al. (1999) found small but systematic group differences between 1st grade dyscalculic children and controls in number naming, number writing and magnitude comparison. Geary, Bow-Thomas, and Yao (1992) found that dyscalculic children are less likely to detect counting errors than control children, and are delayed in understanding the principles of counting (Gelman \& Gallistel, 1978).

However, Russell and Ginsburg (1984) tested children with "mathematics difficulties" on tasks involving 'informal' numerical concepts, including indicating which of two 
numbers is more, indicating which of two numbers is closer to a stated number $x$, and estimation of quantities. Their criterion for mathematics difficulty is three stanines, or one grade below CA on the Iowa Mathematics Achievement Test, with exclusions for low IQ or sensory or motor disabilities. This yields a sample of about $7 \%$ of the test population before attrition, which would be in line with the dyscalculia prevalence estimates, so these subjects seem to be similar to the studies where children are described as "dyscalculic". They found that, despite arithmetical difficulties, these children have normal 'informal' or conceptual understanding. This study is of theoretical importance, since the findings imply that the problems faced by dyscalculic children are specifically to do with memory rather than with numerical understanding. However, very few (between four and six) items were presented for each task; there was a $50 \%$ probability of answering correctly by chance; and error rate, a relatively insensitive measure, was used as the only indicator of performance. As a matter of fact, on the 'which is more' task, all groups performed near ceiling, and on the 'which is closer to $x$ ' task, all groups performed near chance. In an estimation task six items were presented, and while the difference between groups was not statistically significant, it should be noted that there was a difference of nearly an item between the mean scores of control and dyscalculic children. These findings are in need of replication using more items and more sensitive measures such as reaction time latencies.

In conclusion, information regarding dyscalculics' numerical abilities is rather scattered and conflicting, but does indicate that general difficulties with basic number processing may be a feature of dyscalculia. Attempts to discover the root cause of dyscalculia by correlating it with other disabilities have not so far been successful. This approach assumes that number skills are relatively of higher-order and predicated on other abilities such as verbal or spatial skills. However, neuropsychological and developmental research points to the existence of a 'number module' based in the parietal lobe (Butterworth, 1999) for dealing with numerical representations. We propose that the underlying cause of dyscalculia is likely to be related to dysfunction of this system.

\subsection{Dyscalculia as a deficit in the concept of numerosity and its processing}

Although to our knowledge there has been no systematic attempt to investigate specifically the numerical skills of dyscalculic children, studies have consistently found evidence of difficulties even on simple number tasks.

Further examination of the basic number processing abilities of dyscalculic children is necessary for two reasons. First a more fine-grained picture of their difficulties is needed in order to make theoretical headway. If, as proposed here, the deficit is numerical in origin, all aspects of numerical processing are likely to be affected. If it is not, then more information regarding the specific numerical deficits displayed by dyscalculic children will be helpful in relating arithmetic deficits to other underlying abilities. In addition, the utility of subtyping dyscalculics according to neuropsychological or cognitive correlates will not be clear until it has been shown that the different subtypes display qualitatively different patterns of numerical deficit. The current study examines basic numerical processing in detail, and employs a subtyping procedure based on comorbid reading difficulties. It is predicted that dyscalculic children will demonstrate a broad range of 
number processing deficits, but that this pattern will not be affected by the presence of comorbid reading disabilities.

The basic numeric processing skills that are examined in the present study include the socially necessary skills of transcoding between a verbal and an Arabic number code (naming and writing of 1- to 3-digit numbers), but the primary focus is on how well children understand numerosity.

Numerosity is a property of a set and is not, therefore, tied to properties of the individual objects that make up that set. The basic principles derived from the concept of numerosity have been elaborated by Gelman and Gallistel (1978) and Piaget (1952). Piaget stressed an understanding of the kinds of transformation that would and would not affect the numerosity of a set in his "conservation" tasks. Thus, moving objects around would not affect numerosity, while adding or subtracting objects would. He also laid emphasis on understanding that two sets would have the same number if and only if their members could be put in one-to-one correspondence. Gelman and Gallistel focused on the procedures for enumerating sets using counting words, but drew attention also to the kinds of procedural variations that would affect the outcome of the count (one-to-one correspondence with the counting words) and which would not (e.g. which object begins the count, and what kind of object is being counted). One should note also that understanding numerosity implies understanding that each numerosity, say 4 , is distinct, and has a one successor, in this case 5 , and that numerosities are ordered by magnitudethat is 5 has a larger magnitude than 4 .

There is evidence that children are born with a capacity for recognizing and even mentally manipulating small numerosities (Starkey et al., 1990; Wynn, 1992, 1995), and adults seem able to carry out judgements on small numerosities with greater speed and accuracy. In this study, we focused on two tasks that required an understanding of numerosity and the ability to recognize and judge small numerosities, and which did not require high levels of formal mathematical achievement. Indeed, all that was needed, was an ability to understand numerosities up to 9 . We therefore used a simple number comparison, which would test whether children understood number magnitudes, and simple counting skill. Response speed was measured for each of the tasks as deficits in dyscalculic children are not always detected in untimed tasks (Jordan \& Montani, 1997). One adult dyscalculic, "Charles", reported by Butterworth (1999) was abnormally slow on both of these tasks.

In addition to tasks assessing basic numeric processing we also carried out a number of non-numeric tasks for which we do not expect specific deficits in dyscalculia. Number naming speed is contrasted with the non-numerical control task of colour naming presented in exactly the same format. The number comparison task includes a nonnumeric control condition where participants had to compare the physical size of the presented numbers. Phonological short-term and working memory (WISC-III digit span forward and backward) and vocabulary (BPVS) were also assessed. Psychomotor functions were assessed by the WISC-III Mazes subtest which requires adequate visuomotor co-ordination as well as executive functions (planning the way through the maze, monitoring if you are still on the right track and inhibition of going into blind alleys). Although all of these skills were suspected to be deficient in dyscalculia in the literature, 
we predict that the deficits of the dyscalculic children will be confined to numerical processing.

A timed arithmetic test was used rather than scores on an achievement test to classify dyscalculic children. Numerical achievement tests yield a composite score based on a range of different abilities (for example number reading and writing, arithmetic, ability to carry and borrow, spatial and organizational skills) so that it is not clear which abilities are problematic. Achievement test scores may fluctuate over time and may not be reliable. In addition, achievement tests are generally untimed, although evidence indicates that dyscalculic children may perform as well as controls in untimed conditions (Jordan \& Montani, 1997). Ashcraft et al. (1992) found no correlation between scores on a test of mathematical achievement and numerical abilities for their left-lesioned maths disabled subjects. It was felt that a timed arithmetic test would be a more sensitive index of dyscalculia, given that difficulty with arithmetic facts is a defining feature of the disorder.

\section{Method}

\subsection{Participants}

Fifty-four participants were selected from a larger group of 89 4-year-old children from 11 middle schools in the London area. Nine of the schools were state schools, one was a private school specialized for dyslexic children and one was a normal private school. Initial selection was based upon teacher assessment: teachers were asked to nominate children who they felt were of normal intelligence but had serious difficulties with reading, numeracy or both. For each of these children a control child of average ability was tested who was of the same gender and from the same class, in order to minimize as much as possible effects of instruction. Clearly controls could not be matched in this way for the children from the specialized private school, so controls were taken from a nearby private school.

IQ was assessed using Ravens CPM (Raven, Court, \& Raven, 1986). For seven children recent scores from a full WISC-III (Wechsler, 1992) assessment were available, so their WTSC performance IQ scores were used as measures of non-verbal IQ rather than the CPM for these children. (The CPM is highly correlated with WISC-R scores, with correlations up to +0.86 in some studies; Raven et al., 1986.) All children were administered the Number Skills and the Word Reading subscale from the British Ability Scales (BAS II, Elliot, Smith, \& McCulloch, 1997). A customized timed test involving simple addition, subtraction and multiplication was also administered (see Tasks below). Data were discarded if the child was bilingual $(N=4)$ or had been diagnosed with ADHD $(N=2)$.

\subsubsection{Classification scheme}

To qualify for the control group $(N=28)$, children had to have been nominated as 'average' by their teacher, and to have scored between the 25th and 90th percentiles for their age on the BAS subtests and non-verbal IQ. To qualify as 'dyslexic' children had to have been nominated as learning disabled by their teacher, to have scored below 
the 25th percentile on the BAS word-reading subscale, and above the 25 th percentile on non-verbal IQ.

Arithmetic skills were assessed by a timed test described below (Tasks). Median reaction times for each of three tasks (addition, subtraction and multiplication) were calculated for each child. The mean of these medians for each child was then used as a measure of speed on the mental arithmetic tasks overall. The number of errors made on each task was summed, giving a total number of errors per child on the mental arithmetic tasks overall. The group means and standard deviations of the reaction times and errors were calculated for the control group only. Any child scoring more than 3 standard deviations above the control mean on reaction time or error was designated as dyscalculic. Two measures (error and reaction time) were used because it had been noted that children who struggled with mental arithmetic tended to adopt one of the two strategies: they would painstakingly work out each sum on their fingers or by counting, leading to generally accurate answers but extremely long RT latencies; or they would simply guess quickly, leading to inaccurate answers but short RT latencies. A composite score for the facts was used because it was a more conservative criterion than using scores on the individual operations. A composite criterion ensured that children designated as having dyscalculia were either struggling with all three arithmetic operations, or were having severe difficulties with at least one operation.

A cutoff of 3 standard deviations is conservative, but was used to ensure a low incidence of false positives. In addition, all children categorized as dyscalculic had been nominated as having learning difficulties by their teacher. Children who scored between 2 and 3 standard deviations above the mean on either of the facts measures (RT and accuracy) were considered 'borderline' and removed from the analysis.

Three groups were formed on the basis of these criteria: a group of 10 dyslexic children ( 6 boys, 4 girls), who scored below the 25 th percentile on the BAS word-reading task but were within the 2 nd standard deviation above the mean for both arithmetic facts measures (RT and accuracy); a group of 10 dyscalculic children ( 8 boys, 2 girls) who scored above the 25 th percentile on the BAS word-reading and whose performance was more than 3 standard deviations below the mean on at least one of the facts measures; and a group of 11 double deficit (dyslexic and dyscalculic) children ( 8 boys, 3 girls) who scored below the 25 th percentile on the reading test and whose performance was also more than 3 standard deviations below the mean for at least one of the facts measures. Although these groups are small, it was felt that identification of children with serious difficulties was a higher priority than large sample sizes, since patterns of ability may differ between children with genuine learning disabilities and those with relatively minor problems.

Once the three LD groups had been identified, an attempt was made to match the control group as closely as possible to the dyscalculic and dyslexic groups for reading and arithmetic facts, respectively. Ten controls with reading ages a year or more ahead of their chronological ages were discarded, since dyscalculic children tended to be average or somewhat below on the reading test. Thus the matched control group consisted of 18 children ( 8 boys, 10 girls).

The means and standard deviations for the new matched control group for arithmetic facts were checked against the criteria based on the old control group. There was very little difference between the two sets of criteria, except that those based on the new 
Table 1

Subject details

\begin{tabular}{|c|c|c|c|c|}
\hline & $\begin{array}{l}\text { Control } \\
(N=18)\end{array}$ & $\begin{array}{l}\text { Dyslexic } \\
(N=10)\end{array}$ & $\begin{array}{l}\text { Dyscalculic } \\
(N=10)\end{array}$ & $\begin{array}{l}\text { Double deficit } \\
(N=11)\end{array}$ \\
\hline Age (months) & 108.7 (8.6) & $110.1(5.9)$ & $103.7(6.0)$ & $103.9(5.7)$ \\
\hline $\begin{array}{l}\text { Raven CPM } \\
\text { (raw scores) }\end{array}$ & $28.8(3.4)^{\mathrm{a}}$ & $29.7(3.7)$ & $28.5(3.8)^{b}$ & $27.0(3.4)^{\mathrm{c}}$ \\
\hline $\begin{array}{l}\text { BAS reading } \\
\text { (RA-CA in months) }\end{array}$ & $-0.94(6.9)$ & $-19.90(4.8)$ & $-6.30(6.4)$ & $-19.73(6.6)$ \\
\hline $\begin{array}{l}\text { BAS numeracy } \\
\text { (NA-CA in months) }\end{array}$ & $5.72(8.1)$ & $0.90(5.5)$ & $-8.20(10.4)$ & $-7.18(8.3)$ \\
\hline
\end{tabular}

${ }^{\text {a }} N=17$, one child had a WISC performance IQ score.

${ }^{\text {b }} N=8$, two children had WISC performance IQ scores.

c $N=7$, four children had WISC performance IQ scores.

matched group were marginally more lenient, due to reduced variability. However, this difference was not large enough to affect the classifications. The subject details are presented in Table 1.

Age differences between groups did not reach statistical significance; however, dyscalculic and double deficit children were on average 5 months younger than control children and 7 months younger than the dyslexic group. Given that younger children might be expected to be poorer at arithmetic and at number processing generally, age was entered as a covariate into all subsequent analyses.

An independent samples ANCOVA (controlling for age) indicated that there were no differences between groups on the Raven $\operatorname{CPM}(F<1)$. There was a statistically significant difference between groups on Word Reading: $F(3,48)=29.7, P<0.001$. $t$-Tests indicated that the double deficit group had lower scores than controls: $t(27)=7.2, P<0.001$; and dyscalculic children: $t(19)=4.7, P<0.001$. The dyslexic group also scored lower than controls: $t(26)=7.6, P<0.001$; and dyscalculic children: $t(18)=5.4, P<0.001$. There were no differences between the dyscalculic group and controls, or between the double deficit and the dyslexic group. Separate ANCOVAs with age as a covariate were carried out on speed and accuracy of arithmetic facts. There were statistically significant differences between groups for both speed: $F(3,40)=12.2, P<0.001$; and accuracy: $F(3,43)=10.7$, $P<0.001$. Group comparisons controlling for age indicated that the double deficit and the dyscalculic group were both slower and less accurate than the control group and the dyslexic group (all $P<0.05$ ). There were no differences in either speed or accuracy between the double deficit group and the dyscalculic group, or between the dyslexic group and the control group. The BAS Number Skills test was included as a comparison measure, although it was not used for selection. There was a significant difference between groups: $F(3,48)=9.1, P<0.001$. $t$-Tests indicated that the double deficit group had lower scores than controls: $t(27)=4.1, P<0.001$; and lower scores than the dyslexic group: $t(19)=$ $2.7, P=0.01$. The dyscalculic group also had lower scores than controls: $t(26)=3.9$, $P=0.001$; and lower scores than the dyslexic group: $t(18)=2.5, P=0.02$. There was no difference between the double deficit group and the dyscalculic group; or between the dyslexic and the control groups. 
The above analysis involving the two criteria for categorization (word reading and arithmetic facts) indicates that the groups have been classified correctly and are reasonably well matched. The analysis of the BAS Number Skills test is also encouraging, since this measure was not used to categorize the children. This suggests that arithmetic fact ability, at least in our sample, is associated with the global numerical ability tapped by standardized tests.

\subsection{Tasks}

\subsubsection{Standardized tests}

Ravens Coloured Progressive Matrices was administered as a measure of non-verbal reasoning ability, and the British Picture Vocabulary Scale (Dunn, Dunn, \& Whetton, 1997) as a measure of verbal ability. The Word Reading scale from the British Ability Scale (BAS II) was used as measure of reading ability. The Number Skills scale from the BAS was also administered. Phonological short-term and working memories were measured using the Digit Span scale from the WISC-III, which includes forwards and backwards digit span. Finally, the Mazes subscale from the WISC-III was used.

\subsubsection{Computer tasks}

The computer tasks were presented on a Macintosh PowerBook running SuperLab software. Unless otherwise specified, numbers were $0.6 \times 1 \mathrm{~cm}$ in size, presented in an $8 \times 7 \mathrm{~cm}$ field in the centre of the screen. Items were presented after a $400 \mathrm{~ms}$ fixation point accompanied by an acoustic signal. Responses were verbal and triggered a voiceactivated key which measured reaction latencies from the onset of presentation. All items remained visible until the voice key was activated. The experimenter pressed a key in order to trigger the next item.

Mental arithmetic. 12 simple additions, 12 simple subtractions and 12 simple multiplications were presented in 3 separate blocks. All involved single-digit numbers from 2 to 9 , excluding 0 and 1 , since number facts involving 0 and 1 can be solved by application of a rule rather than calculation or retrieval. No ties (e.g. $3+3,5 \times 5)$ were presented, and items were not repeated. Items were presented on the computer screen in the form " $3+4$ ". Six practice addition sums were given before the first block (additions); four practice subtractions before the start of the subtraction block, and four practice multiplications before the start of the multiplication block. Children were asked to say the answer as quickly as they could without making any mistakes. They were also told that if they didn't already know the answer, they could use whatever way they found easiest to work it out. Reaction latencies were recorded using a voice-activated key, and errors were recorded by the experimenter.

\subsection{Basic number processing skills}

\subsubsection{Number reading and naming}

1- and 2-digit numbers were presented individually on the computer. Children were asked to name the numbers as quickly as they could without making any mistakes, and response latencies were recorded using a voice-activated key. Errors were recorded by 
the experimenter. Six practice items were given, followed by a block of 18 single-digit items (each of the numbers from 1 to 9 appeared twice in a pseudo-random order with the proviso that no item appeared twice in succession). A block of 16 2-digit items was then presented. Numbers were between 20 and 90, avoiding teens.

Colour naming was used as a non-numerical control for the number naming tasks. Rectangles of five different colours (red, green, brown, blue and black) were presented on the computer. Each colour was presented four times in pseudo-random order with the proviso that no item appeared twice in succession. Five practice items were given. Response latencies were measured using a voice key, and errors were noted by the experimenter.

A list of eight 3-digit numbers was also presented. These numbers were presented simultaneously on a sheet of paper, and children were asked to read them as quickly as they could without making any mistakes. A stopwatch was used to measure the time taken by the child to read the whole list. Simultaneous presentation was used for this task in order to record the amount of time taken to articulate 3-digit numbers: a voice key measures only speech onset.

\subsubsection{Number comparison}

Children were presented with two digits (1-9, not including 5) on the computer, one to the left and one to the right of the screen. The digits could vary along two dimensions: numerical size and physical size. In one variant of the task children were asked to select the numerically largest number, in the other variant they were asked to select the physically largest number. Congruence between physical and numerical size was counterbalanced; so that there were 12 congruent trials, 12 neutral trials (in which either the numerical or the physical size of the two numbers was the same) and 12 incongruent trials for each variant of the task. There were two numerical size differences: 1 (e.g. 2, 3) and 5 (e.g. 7,2$)$ and two physical sizes: $0.3 \times 0.5 \mathrm{~cm}$, for small numbers, and $0.6 \times 1 \mathrm{~cm}$ for large numbers. Items were presented in a pseudo-random order. The numbers were presented in an $11 \times 8.5 \mathrm{~cm}$ field in the centre of the screen. Each item was preceded by a fixation cross that lasted for $300 \mathrm{~ms}$. Response keys were ' $\mathrm{f}$ ' and ' $\mathrm{J}$ ' on the computer keyboard. Children were asked to hit the key to the same side (left or right) to the side where the larger number had appeared. Reaction times and errors were recorded by the computer.

Order of presentation of the two variants of the task was counterbalanced as far as possible, each LD child being presented with the opposite order to the last, and each control child being presented with the same order as the matched LD child.

\subsubsection{Number writing}

The experimenter dictated a number to the child who immediately wrote it on a piece of paper. 10 single-digit numbers (in random order); eight 2-digit numbers and eight 3-digit numbers were presented. Errors were scored as substitutions, reversals, or place-value errors. An error was scored as a substitution if an alternative digit was substituted for the correct one (e.g. 26 written as 22). Reversals were when a digit was written the wrong way around (e.g. 3 written as $\varepsilon$ ). Place value errors involved syntax confusion (e.g. 724 written as 7024). 


\subsubsection{Number sequences/counting}

Children were asked to count as quickly as they could from 1 to $20 ; 45$ to $65 ; 2$ to 20 in twos; and 20 to 1 backwards. Each counting task was timed using a stopwatch from the first to the last item. Errors were noted.

\subsubsection{Dot counting}

Groups of randomly arranged dots ranging from 1 to 10 were presented on the computer. Children were asked to count the dots and to respond as quickly as they could without making any mistakes. A voice key recorded reaction times. Errors were recorded by the experimenter. 20 trials were presented altogether, with each number from 1 to 9 being represented twice in a pseudo-random order with the proviso that no item occurred twice in succession.

\subsubsection{Procedure}

Head teachers and special needs co-ordinators were contacted either directly or via their Local Education Authority. Those who agreed to take part in the study asked teachers in their school to nominate children according to the criteria, and sent consent forms to parents. Children were tested individually in a quiet room in their school. Testing was done as far as possible in a single session, otherwise it was completed the next day. Sessions generally lasted from 1 to $2 \mathrm{~h}$, depending on the ability of the child.

\section{Results}

The data were analysed using separate ANCOVAs (with age as a covariate) for each task. When indicated by statistically significant effects in the main analysis, planned comparisons (also controlling for age) were carried out between the control group and the two groups with number fact deficits (dyscalculic and double deficit), between the control group and the dyslexic group and between the dyscalculic group and the double deficit group.

\subsection{Standardized tests}

Mean scores and standard deviations for the standardized tests are presented in Table 2.

Digit span. In both conditions (forward and backward), Table 2 shows a trend of lower performance of the two reading disabled groups (dyslexic and double deficit) compared to the two other groups. However, in a repeated measures ANCOVA (controlling for age) the group effect did not reach statistical significance $(F(3,38)=2.5, P=0.08)$, and neither did the interaction between group and condition $(F(3,38)=0.1, P>0.1)$.

Mazes. There was also no statistically significant difference between groups on the Mazes.

Vocabulary. With most children, the British Picture Vocabulary Test (Dunn et al., 1997) could be carried out. In an ANCOVA controlling for age no group differences could be found. For 7 children ( 1 control, 2 dyscalculic and 4 double deficit children) recent scores of the WISC-vocabulary scales were available so that carrying out the BPVS was 
Table 2

Mean scores (standard deviations) for standardized tests

\begin{tabular}{lcccc}
\hline & $\begin{array}{l}\text { Control } \\
(N=18)\end{array}$ & $\begin{array}{l}\text { Dyslexic } \\
(N=10)\end{array}$ & $\begin{array}{l}\text { Dyscalculic } \\
(N=10)\end{array}$ & $\begin{array}{l}\text { Double deficit } \\
(N=11)\end{array}$ \\
\hline $\begin{array}{l}\text { Digit span forward } \\
\text { (raw score) }\end{array}$ & $8.8(1.5)$ & $7.7(0.7)$ & $8.7(2.6)$ & $7.6(1.1)$ \\
$\begin{array}{l}\text { Digit span backward } \\
\text { (raw score) }\end{array}$ & $4.5(1.4)$ & $3.7(1.1)$ & $4.3(1.3)$ & $3.6(1.5)$ \\
$\begin{array}{l}\text { Mazes (raw score) } \\
\begin{array}{l}\text { Vocabulary } \\
\text { (BPVS raw scores) }\end{array}\end{array}$ & $\begin{array}{l}18.1(3.6) \\
88.1(14.3)^{\mathrm{a}}\end{array}$ & $\begin{array}{l}19.0(2.3) \\
89.3(12.8)\end{array}$ & $\begin{array}{l}18.7(3.8) \\
91.3(3.9)^{\mathrm{b}}\end{array}$ & $\begin{array}{l}78.0(3.8) \\
78.6(13.5)^{\mathrm{c}}\end{array}$ \\
\hline
\end{tabular}

${ }^{\text {a }} N=17$, one child had a WISC vocabulary score.

b $N=8$, two children had WISC vocabulary scores.

c $N=7$, four children had WISC vocabulary scores.

superfluous. All of these children had scaled scores above 10, once again confirming that there were no serious verbal deficits.

\subsubsection{Mental arithmetic}

Errors. A repeated measures ANCOVA, controlling for age, was performed on errors with each operation (addition, subtraction and multiplication) as a separate level. There was a significant effect of level, $F(2,86)=6.2, P=0.003$. Examination of the mean scores presented in Fig. 1 indicates that all groups were less accurate with multiplication than with addition or subtraction. There was also an interaction

Arithmetic facts: accuracy

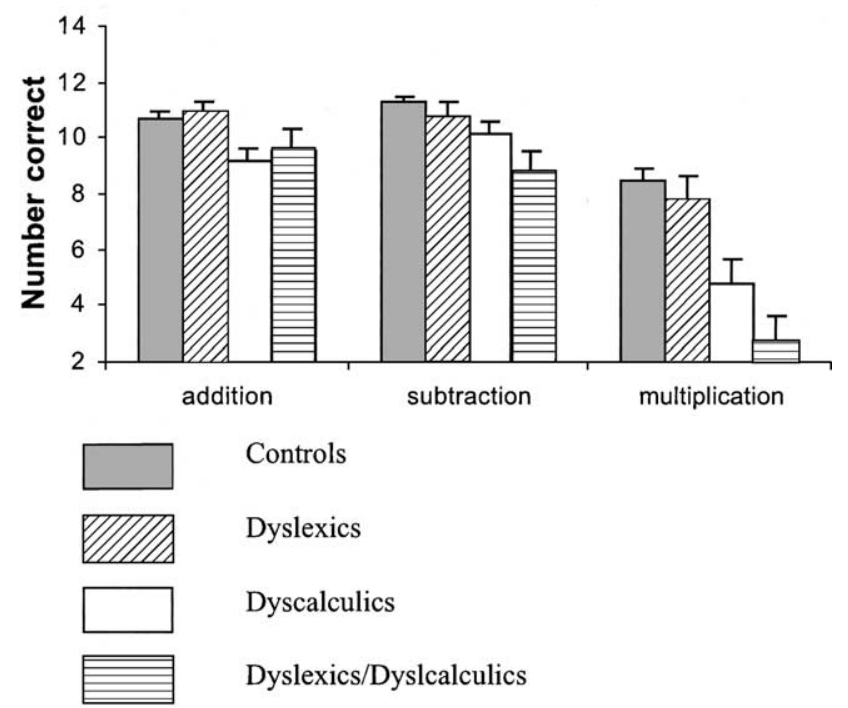

Fig. 1. Mental arithmetic: mean number (SE) of correct responses for each operation. 
between level and group, $F(6,86)=3.2, P=0.007$. Examination of the mean scores for the groups suggests that the double deficit and dyscalculic groups had relatively greater difficulty with multiplications than the other two groups. As expected there was a difference between groups, $F(3,43)=10.7, P<0.001$. Planned contrasts indicated that the double deficit group were less accurate than controls on subtraction and multiplication $(P<0.001)$ but not addition. The dyscalculic group was less accurate than controls on addition and subtraction $(P=0.01)$ and multiplication $(P<$ $0.001)$. No reliable differences were found between dyslexic and control groups and between dyscalculic and double deficit groups.

$R T s$. The median reaction time (for correct answers only) was calculated for each child. As response accuracy was high for additions and subtractions, the median RTs for these conditions are on average based on a reasonable number of 10 responses per child. The average number of correct responses - and therefore the number of RTs was clearly lower. Three children (all from the double deficit group) failed to answer any multiplication questions correctly and had no valid reaction time for multiplication. In order not to lose them from the repeated measures ANCOVA (controlling for age and with addition, subtraction and multiplication as separate levels) their multiplication RT was replaced by the mean score for the double deficit group.

First inspection of the descriptive statistics (see Fig. 2) showed that the variability was high, especially for the dyscalculic and double deficit groups. Therefore, the statistical analysis was carried out on log transformed scores. There was no statistically significant main effect of level and no interaction between level and group. As expected, there was a significant difference between groups, $F(3,43)=12.2$,

Arithmetic facts: RTs
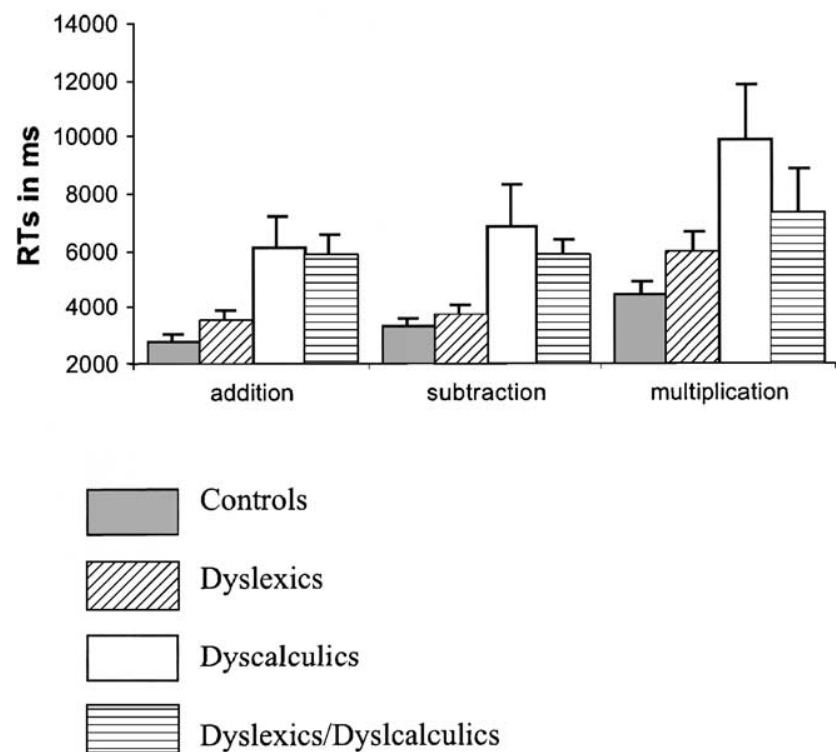

Fig. 2. Mental arithmetic: mean RTs (SE) for correct responses for each operation. 
$P<0.001$. The double deficit group was slower than controls on addition and subtraction $(P<0.001)$ and multiplication $(P=0.03)$. The dyscalulic group was also slower than controls on all operations $(P<0.005)$. The dyslexic group was not statistically different from controls and the dyscalculic group was not statistically different from the double deficit group.

\subsection{Basic number processing skills}

\subsubsection{Number naming and reading}

There was no attempt at statistical analysis of errors for these tasks, due to the very low proportion of errors made.

Median reaction times for correct answers were calculated for each child for 1-digit number naming, 2-digit number naming, and colour naming (see Fig. 3). As response accuracy was high, these median RTs are based on a reasonable number of responses in each condition (18 1-digit numbers, 162 -digit numbers and 20 colour patches). A repeated measures ANCOVA controlling for age was performed on the data, with 1-digit number naming, 2-digit number naming and colour naming as separate levels. There was no main effect of level, and no effect of group. However, there was a statistically significant interaction between level and group: $F(6,0.88)=3.1, P=0.009$. Examination of the mean scores suggested that while the dyslexic group was generally slower at naming than controls, the dyscalculic and double deficit groups appeared to have a particular problem with 2-digit number naming.

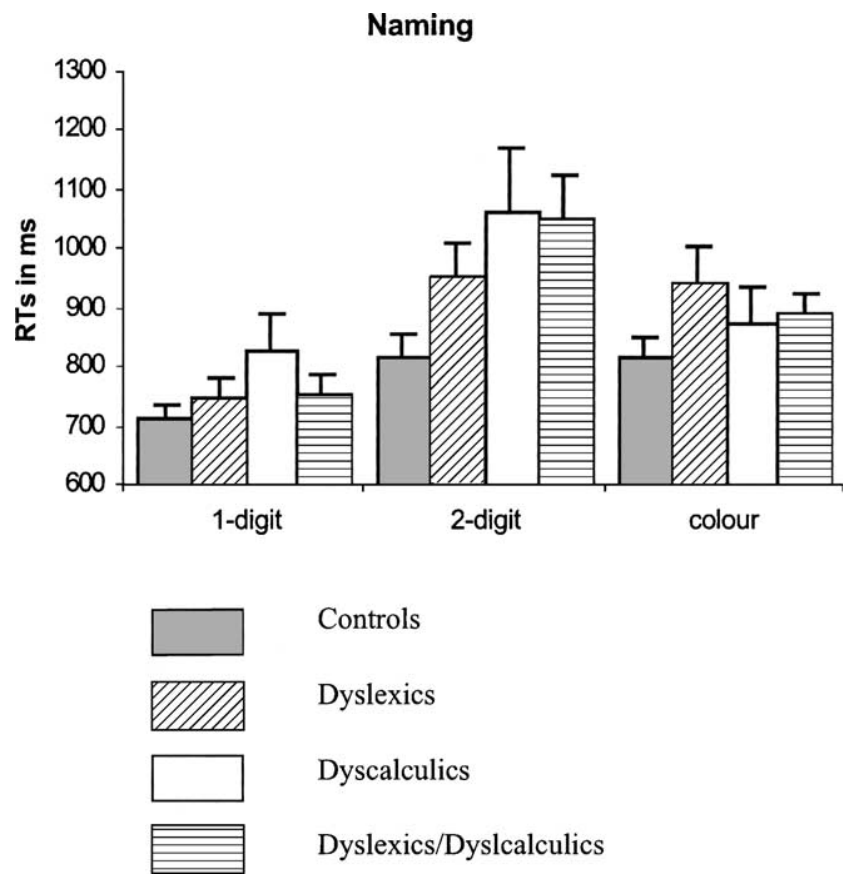

Fig. 3. Naming: mean RTs (SE) for 1- and 2-digit numbers and colours. 
This interaction was investigated further by reanalysing the number naming data with colour naming entered as a covariate. This analysis compares number naming speed across groups whilst controlling for factors such as speed of processing, articulation and access to semantic memory (processes which are also involved in colour naming). A repeated measures ANCOVA with 1- and 2-digit number naming as separate levels and colour naming and age as covariates was performed. Now the only significant effect was group: $F(3,43)=3.3, P=0.03$. Contrasts between each group and the control group indicated that the double deficit and the dyscalculic group were slower at number naming than the control group $(P<0.05)$, even with colour naming controlled. The dyslexic group, however, was no different from controls on number naming once general naming ability was controlled. In a final planned contrast it was confirmed that the dyscalculic group did not differ from the double deficit group.

From the analysis of reading a list of eight 3-digit numbers as quickly as possible one child from the dyscalculic group was excluded due to missing data. A one-way ANCOVA controlling for age was performed on the reading times taken. There was a statistically significant difference between groups: $F(3,47)=4.2, P=0.01$. Contrasts $(P<0.05)$ indicated that the double deficit group $(M=20.3 \mathrm{~s}, \mathrm{SD}=4.6)$ and the dyscalculic group $(M=20.7 \mathrm{~s}, \mathrm{SD}=6.7)$ did not differ from each other but both showed higher number reading times than controls $(M=15.0 \mathrm{~s}, \mathrm{SD}=4.1)$. The dyslexic group $(M=17.8 \mathrm{~s}$, $\mathrm{SD}=4.5)$ performed not reliably different from the control group.

\subsubsection{Number comparison}

Median reaction times for correct answers were calculated for each child for number comparison and size comparison (Fig. 4). A repeated-measures ANCOVA controlling for age was performed on the data. There was a main effect of task (numerical or physical): $F(1,43)=4.1, P=0.05$. Comparison of the means indicates that physical comparison was faster than numerical comparison. There was also an interaction between condition and group: $F(3,43)=6.0, P=0.002$. Contrasts indicated that the double deficit and the dyscalculic group did not differ from each other in any condition but were both slower than controls on the numerical comparison task $(P<0.05)$ though not the physical comparison task. The dyslexic group did not differ from controls on either task. An analysis of the errors found no effect of task, and no differences between groups.

In the numerical condition an effect of congruency of physical and numerical size could be observed $(F(1,43)=6.0, P=0.02)$, however, this effect did not interact with group. Planned comparisons showed that RTs for congruent items (1186 ms) were lower than for neutral $(1340 \mathrm{~ms}, t(47)=5.1, P<0.001)$ and incongruent items $(1362 \mathrm{~ms}, t(47)=8.0$, $P<0.001)$. For the physical condition no effects of congruency were found. In the numerical condition, RTs for items with a larger numerical difference (1199 ms) were lower than for items with a smaller numerical difference (1415), however, this difference was not reliable and did not interact with group.

\subsubsection{Number writing}

Nine children committed one or more substitution errors. Four were from the double deficit group (36\%); 2 from each of the dyscalculic and dyslexic groups (20\%) and 1 from 

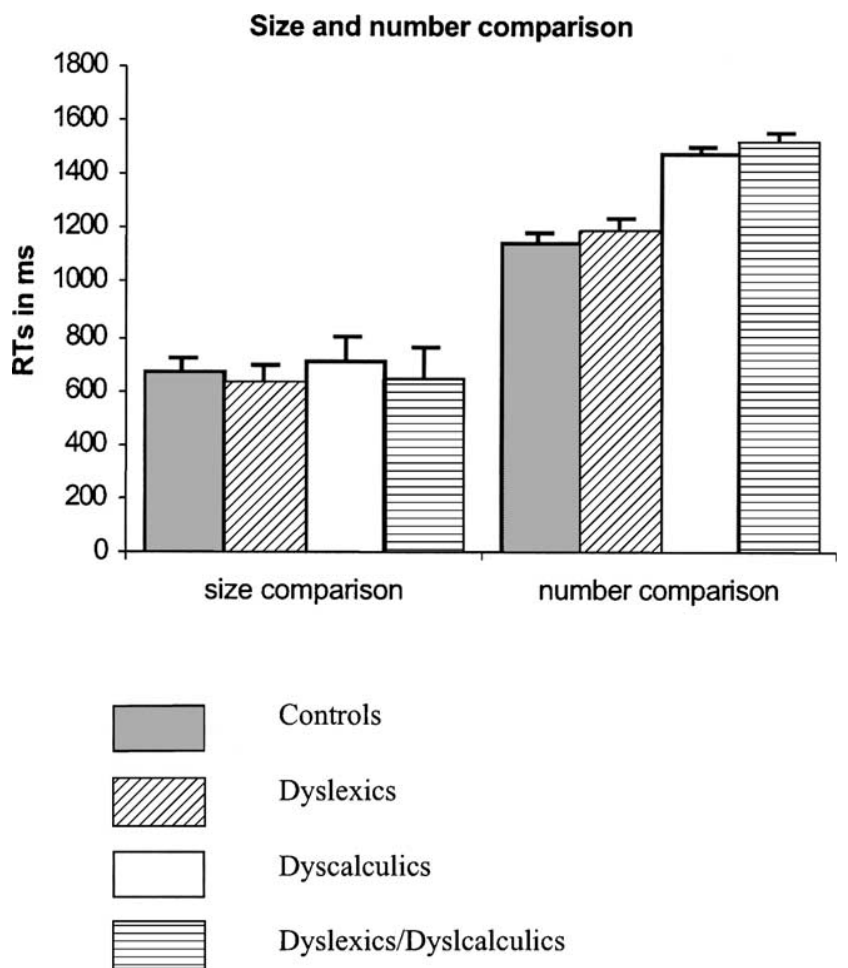

Controls

Dyslexics

Dyscalculics

Dyslexics/Dyslcalculics

Fig. 4. Size and number comparison: mean RTs (SE) for correct responses.

the control group (6\%). An independent samples ANCOVA was performed on the substitutions but did not reach statistical significance.

No statistical analysis was performed on reversals and place value errors, due to the fact that no errors of these types were made by any of the children in the control or dyslexic groups. Variance was thus far from equal between groups. 11 children in total reversed numbers; of these 5 were in the double deficit group (45\%), 6 in the dyscalculic group $(60 \%)$. Five children made place value errors: 1 was in the dyscalculic group (10\%) and 4 were in the double deficit group (36\%).

\subsubsection{Number sequences/counting}

One dyscalculic child was excluded from this analysis because of missing data. A repeated-measures ANCOVA controlling for age was performed on the times taken by each child to count in the four conditions: from 1 to 20 , from 45 to 65 , from 2 to 20 in twos, and from 20 to 1 backwards (see Fig. 5). There was no main effect of counting condition, but there was an effect of group: $F(3,43)=7.0, P=0.001$; and an interaction between group and condition: $F(9,129)=2.3, P=0.021$. Contrasts indicated that the double deficit and the dyscalculic group did not differ from each other and were both slower than controls in all conditions $(P<0.007$ except for dyscalculics in the $1-20$ condition when $P=0.01)$. There was a trend for dyslexic children to be slower at counting although this 


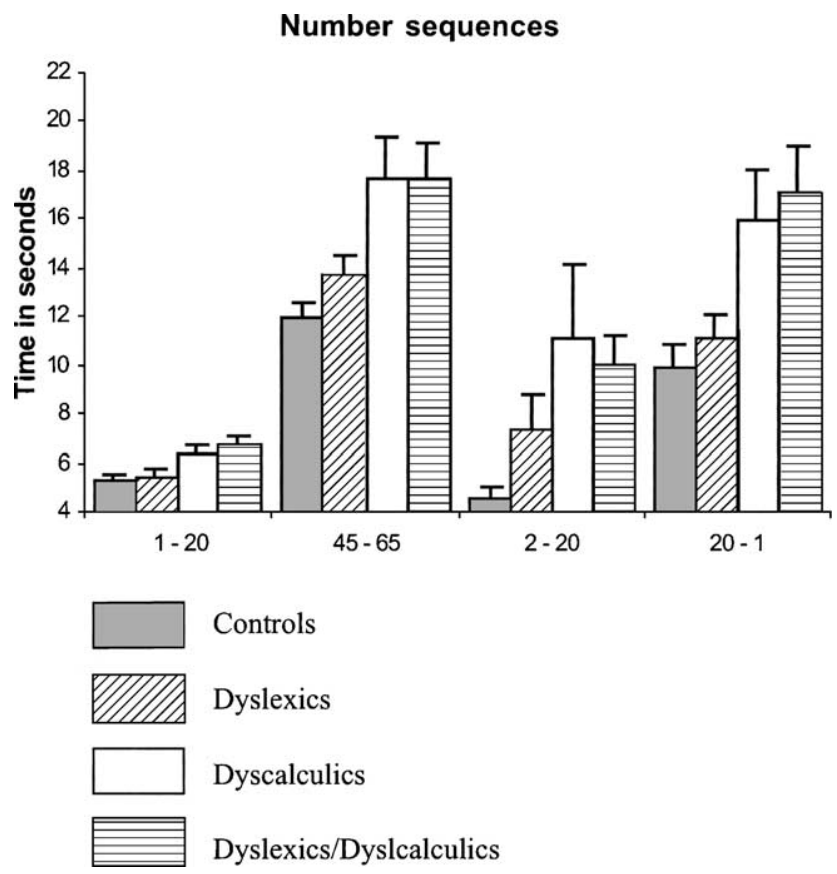

Fig. 5. Number sequences: mean response times (SE) for counting from 1 to $20,45-65,2-20$ in twos and 20-1 backwards.

only reached statistical significance in two conditions: counting $2-20$ in $2 \mathrm{~s}(P=0.02)$ and counting from 45 to $65(P=0.04)$.

\subsubsection{Dot counting}

Only $7 \%$ of all responses were miscounts, almost without exception for six or more dots, deviating from the correct number of dots by one or two. In a non-parametric Kruskal-Wallis test, no group differences were found.

Altogether, the analysis of RTs for correct responses is based on 847 RTs, 17 responses per child on average. Reliability proved to be high with 0.82 . A first inspection of the mean scores showed that for the low range of one to three dots comparably small differences between RTs could be observed while from four dots on a larger and systematic increase of RTs was evident for increasing dot numbers. This was taken as indication for a subitizing range (1-3 dots) and a counting range (4-10 dots). For theoretical reasons and because the distribution of RTs for these two ranges were very different, subitizing range and counting range were analysed separately. For both ranges, the best fitting regression lines were calculated for each child. Fig. 6 presents the mean regression lines for each group for the subitizing and the counting range.

ANCOVAs with age and colour naming as covariates were carried out on the slopes and intercepts of both ranges. Colour naming was introduced as a covariate to control for general differences in naming speed. Fig. 6 shows that within the subitizing range, the slopes for the dyscalculic, and the double deficit group are steeper than those of 

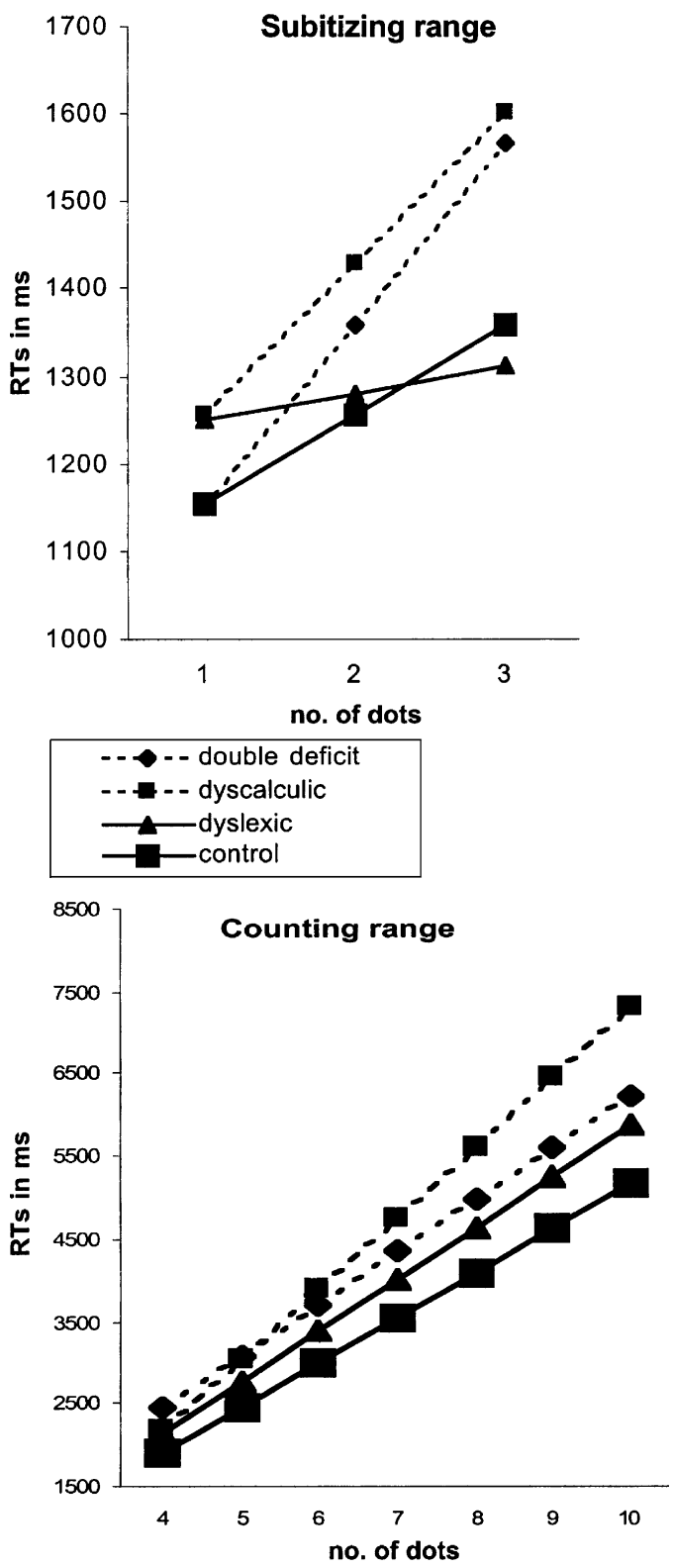

Fig. 6. Dot counting: regression lines for subitizing and counting range.

the dyslexic and the control groups; however, this was not statistically reliable. For the counting range, the group difference was marginally significant $(F(3,47)=2.7$, $P=0.06)$. Fig. 6 indicates that this is due to a steeper slope of dyscalculic compared to control children. There was no difference between the intercepts of the four groups, neither for subitizing nor for counting range. 


\section{General discussion}

In summary, the dyscalculic children identified in this study demonstrated general deficits in number processing, including accessing verbal and semantic numerical information, counting dots, reciting number sequences and writing numbers. Despite this, dyscalculic children without reading disability were normal or above average on tasks involving phonological working memory, accessing non-numerical verbal information, non-verbal intelligence, language abilities, and psychomotor abilities. As predicted, this pattern of deficits is at once too broad and too specific to be readily explainable in terms of general spatial, verbal or memory abilities. In terms of these results, dyscalculia can best be defined as a deficit in the representation or processing of specifically numerical information.

Neuropsychological evidence indicates that numerical processing is localized to the parietal lobes bilaterally, in particular the intra-parietal sulcus (Dehaene, Piazza, Pinel, \& Cohen, 2003), and is independent of other abilities. Developmental dyscalculia is likely to be the result of the failure of these brain areas to develop normally, whether because of injury or because of genetic factors.

Children with reading disability only performed similarly to controls on the numerical tasks. They were slower than controls in reciting number sequences (although less so than dyscalculic children) and there were non-significant trends towards slowness in number reading and number naming. However, unlike with the dyscalculic groups, the number naming trend disappeared once general naming ability was controlled for. Dyslexic children were also identical to controls on non-verbal (or non-phonological) tasks such as number writing and number comparison. This pattern of results suggests that children with reading difficulties only do not have number processing deficits, although difficulties with verbal or phonological aspects of some of these tasks may have affected their performance.

The patterns of performance of the two dyscalculic groups on the numerical tasks were very similar. This study has found no evidence for a qualitative difference in the numerical abilities of dyscalculic children with and without reading disabilities. In many tasks the double deficit group's performance was slower or more error-prone than that of the dyscalculic group, suggesting that their difficulties may be more severe; this is in keeping the findings of Jordan and Montani (1997) and Shalev et al. (1997). However, the pattern of impairment was the same for both groups: they each appeared to struggle with every aspect of numerical processing tested in this study. This finding is further evidence against theories which posit differential causes for different subgroups of dyscalculic children.

There are interesting parallels with and divergences from recent studies by Jordan and colleagues (Jordan et al., 2003a,b) of second and third grade children using "achievement groups" with similar names to our own: "math difficulties", "math and reading difficulties", "reading difficulties", and "normal achievement". However, their groups were defined by the 35th percentile, a far more inclusive criterion than the highest estimates for dyscalculia prevalence (see above for a discussion). Their affected groups would therefore include children whose mathematics difficulties are not severe enough to fall outside the usual definition of the average range, as well as some proportion of dyscalculics as determined by more stringent criteria, and a large proportion of children whose maths achievement is likely to be poor for a wide variety of reasons. 
One important finding of Jordan et al.'s studies is that the growth curves of performance on mathematics achievement tasks are similar for all groups. This is in contrast with the findings that dyscalculia is a persistent deficit involving the maintenance of immature strategies, which causes dyscalculic children to fall farther and farther behind their peers (e.g. Ostad, 1998, p. 359; Ostad, 1999, p. 360).

A second important finding was that there were no differences between groups with and without reading difficulties on fact retrieval—which supports our general claim about the functional independence of learning to read and learning arithmetic, and that fact retrieval is not, in essence, a verbally mediated process. Indeed, they write that "our data do not support the suggestion that difficulties in reading and fact retrieval share a core-underlying deficit related to phonological processing”. (Jordan et al., 2003a, p. 24)

In five of their seven tasks, IQ "added little information beyond our initial group classification" (p. 26) reinforcing our own conclusion that low general cognitive ability is neither necessary nor sufficient to cause dyscalculia.

We suggest that the key deficit in developmental dyscalculia is a failure to represent and process numerosity in a normal way. Numerical expressions do not seem to have the same meaning for these children, as is evidenced by the relative difficulty they have with the number comparison and dot counting. Failure to develop normal representations may account for the difficulty experienced by dyscalculic children in memorizing arithmetical facts: these facts lack meaning for them, or, at least, they do not carry the usual systematic meanings that make for well-ordered and accessible memories. This account would be consistent with the finding that there are no general short-term or long-term memory deficits observed in the DC children. However, this proposal requires further investigation.

In conclusion, the most likely candidate for an underlying cause of dyscalculia is a congenital failure to understand basic numerical concepts, especially the idea of numerosity, a capacity which is independent of other abilities. This is revealed by deficits in very basic numerical capacities, dot counting, and number comparison; there was also a trend towards a difference in subitizing. This study has demonstrated that dyscalculic children without verbal or psychomotor difficulties have a range of numerical difficulties relative to controls. (The small sample-size defined by tight criteria means that the possibility cannot be excluded that there are mathematical difficulties with a different cognitive basis.)

We suggest that lack of understanding of numerosity, and a poor capacity to recognize and discriminate small numerosities - as revealed in performance on dot tasks-may prevent dyscalculics developing the normal meanings for numerical expressions and lead to their difficulties in learning and retaining information regarding numbers. Geary (1993) proposes that dyscalculic children may well have trouble with representation of numbers in semantic memory, although we would argue that this does not imply anything about their semantic memory for anything else.

We suggest that future research on dyscalculia should focus upon the numerical basis of dyscalculia rather than upon its correlates in other cognitive domains. The study described here was based upon small groups of children, and the definition of "dyscalculia" that we used was based upon difficulties specifically with number facts. Further research may identify other subgroups of dyscalculic, who may display other patterns of disability. 
However, on the basis of the evidence so far, numerical abilities should take centre stage in further research into the nature of dyscalculia.

\section{Acknowledgements}

This research was supported by the Department for Education and Skills SEN Small Programmes grant to UCL and the British Dyslexia Association and an APART grant of the Austrian Academy of Science to Karin Landerl. We thank Dr Lindsay Peer of the British Dyslexia Association and Eva-Maria Ebner for their contributions to this project. We are grateful to the students and teachers of the participating schools in the London Boroughs of Harrow and Camden.

\section{References}

American Psychiatric Association (1994). Diagnostic and statistical manual of mental disorders (4th ed). Washington, DC: American Psychiatric Association.

Ashcraft, M. H., Yamashita, T. S., \& Aram, D. M. (1992). Mathematics performance in left and right brainlesioned children and adolescents. Brain and Cognition, 19, 208-252.

Baddeley, A. D., Lewis, V., \& Vallar, G. (1984). Exploring the articulatory loop. Journal of Experimental Psychology, 36A, 233-252.

Badian, N. A. (1983). Arithmetic and nonverbal learning. In H. R. Myklebust (Ed.), (Vol. 5) (pp. 235-264). Progress in learning disabilities, New York: Grune and Stratton.

Bisanz, J. (1999a). The development of mathematical cognition: Arithmetic. Journal of Experimental Child Psychology, 74, 153-156.

Butterworth, B. (1999). The mathematical brain. London: Macmillan.

Butterworth, B., Cipolotti, L., \& Warrington, E. K. (1996). Short-term memory impairments and arithmetical ability. Quarterly Journal of Experimental Psychology, 49A, 251-262.

Butterworth, B., Grana, A., Piazza, M., Girelli, L., Price, C., \& Skuse, D. (1999). Language and the origins of number skills: karyotypic differences in Turner's syndrome. Brain and Language, 69(3), 486-488.

Cappelletti, M., Butterworth, B., \& Kopelman, M. D. (2001). Spared numerical abilities in a case of semantic dementia. Neuropsychologia, 39/11, 1224-1239.

Cohen, L., Dehaene, S., Cochon, F., Lehericy, S., \& Naccache, L. (2000). Language and calculation within the parietal lobe: A combined cognitive, anatomical and fMRI study. Neuropsychologia, 38, 1426-1440.

Dehaene, S., Dehaene-Lambertz, G., \& Cohen, L. (1998). Abstract representations of numbers in the animal and human brain. Trends in Neurosciences, 21(8), 355-361.

Dehaene, S., Piazza, M., Pinel, P., \& Cohen, L. (2003). Three parietal circuits for number processing. Cognitive Neuropsychology, 20, 487-506.

Dunn, L. M., Dunn, L. M., \& Whetton, C. (1997). The British picture vocabulary scales (2nd ed.). Windsor: NFER-Nelson.

Elliot, C., Smith, P., \& McCulloch, K. (1997). British ability scales II. Windsor: NFER-Nelson.

Fayol, M., Barrouillet, P., \& Marinthe, C. (1998). Predicting arithmetical achievement from neuro-psychological performance: A longitudinal study. Cognition, 68, 63-70.

Fletcher, J. F. (1985). Memory for verbal and nonverbal stimuli in learning disabled subgroups: Analysis by selective reminding. Journal of Experimental Child Psychology, 40, 244-259.

Gallistel, C. C. (1990). The organization of learning. Cambridge, MA: MIT Press.

Geary, D. C. (1993). Mathematical disabilities: Cognition, neuropsychological and genetic components. Psychological Bulletin, 114, 345-362. 
Geary, D. C., Bow-Thomas, C. C., \& Yao, Y. (1992). Counting knowledge and skill in cognitive addition: A comparison of normal and mathematically disabled children. Journal of Experimental Child Psychology, 54, $372-391$.

Geary, D. C., Hamson, C. O., \& Hoard, M. K. (2000). Numerical and arithmetical cognition: A longitudinal study of process and concept deficits in children with learning disability. Journal of Experimental Child Psychology, 77, 236-263.

Geary, D. C., \& Hoard, M. K. (2001). Numerical and arithmetical deficits in learning-disabled children: Relation to dyscalculia and dyslexia. Aphasiology, 15(7), 635-647.

Geary, D. C., Hoard, M. K., \& Hamson, C. O. (1999). Numerical and arithmetical cognition: Patterns of functions and deficits in children at risk for a mathematical disability. Journal of Experimental Child Psychology, 74, 213-239.

Gelman, R., \& Gallistel, C. R. (1978). The child's understanding of number (1986 ed.). Cambridge, MA: Harvard University Press.

Gerstmann, J. (1940). Syndrome of Finger Agnosia: disorientation for right and left, agraphia and acalculia. Archives of Neurology and Psychiatry, 44, 398-408.

Ginsburg, H. P. (1997). Mathematics learning disabilities: A view from developmental psychology. Journal of Learning Disabilities, 30, 20-33.

Hanich, L. B., Jordan, N. C., Kaplan, D., \& Dick, J. (2001). Performance across different areas of mathematical cognition in children with learning difficulties. Journal of Educational Psychology, 93, 615-626.

Jordan, N., Hanich, L. B., \& Kaplan, D. (2003a). A longitudinal study of mathematical competencies in children with specific mathematics difficulties versus children with co-morbid mathematics and reading difficulties. Child Development, 74, 834-850.

Jordan, N., Hanich, L. B., \& Kaplan, D. (2003b). Arithmetic fact mastery in young children: A longitudinal investigation. Journal of Experimental Child Psychology, 85, 103-119.

Jordan, N., Kaplan, D., \& Hanich, L. B. (2002). Achievement growth in children with learning difficulties in mathematics: Findings of a two-year longitudinal study. Journal of Educational Psychology, 94, 586-597.

Jordan, N., \& Montani, T. (1997). Cognitive arithmetic and problem solving: A comparison of children with specific and general mathematics difficulties. Journal of Learning Disabilities, 30(6), 624-634.

Kinsbourne, M., \& Warrington, E. K. (1963). The developmental Gerstmann Syndrome. Annals of Neurology, 8, 490-501.

Kirby, J. R., \& Becker, L. D. (1988). Cognitive components of learning problems in arithmetic. Remedial and Special Education, 9, 7-16.

Koontz, K. L., \& Berch, D. B. (1996). Identifying simple numerical stimuli: Processing inefficiencies exhibited by arithmetic learning disabled children. Mathematical Cognition, 2(1), 1-23.

Lewis, C., Hitch, G., \& Walker, P. (1994). The prevalence of specific arithmetic difficulties and specific reading difficulties in 9- and 10-year old boys and girls. Journal of Child Psychology and Psychiatry, 35, $283-292$.

McLean, J. F., \& Hitch, G. J. (1999). Working memory impairments in children with specific arithmetical difficulties. Journal of Experimental Child Psychology, 74, 240-260.

Ostad, S. E. (1998). Comorbidity between mathematics and spelling difficulties. Logopedics Phoniatrics Vocology, 23, 145-154.

Ostad, S. E. (1999). Developmental Progression of subtraction studies: a comparison of mathematically normal and mathematically disabled children. European Journal of Special Needs Education, 14(1), 21-36.

Piaget, J. (1952). The child's conception of number. London: Routledge \& Kegan.

Raven, J. C., Court, J. H., \& Raven, J. (1986). Manual for Raven progressive matrices and vocabulary scales. London: Lewis.

Rosenberg, P. B. (1989). Perceptual-motor and attentional correlates of developmental dyscalculia. Annals of Neurology, 26, 216-220.

Rourke, B. (1989). Nonverbal learning disabilities. The syndrome and the model. New York: Guilford Press.

Rourke, B. P. (1993). Arithmetic disabilities, specific and otherwise: A neuropsychological perspective. Journal of Learning Disabilities, 26, 214-226.

Russell, R. L., \& Ginsburg, H. P. (1984). Cognitive analysis of children's mathematical difficulties. Cognition and Instruction, 1, 217-244. 
Shalev, R. S., \& Gross-Tsur, V. (1993). Developmental dyscalculia and medical assessment. Journal of Learning Disabilities, 26, 134-137.

Shalev, R. S., \& Gross-Tsur, V. (2001). Developmental dyscalculia. Review article. Pediatric Neurology, 24, $337-342$.

Shalev, R. S., Manor, O., \& Gross-Tsur, V. (1997). Neuropsychological aspects of developmental dyscalculia. Mathematical Cognition, 3(2), 105-120.

Siegel, L. S., \& Feldman, W. (1983). Non-dyslexic children with combined writing and arithmetic difficulties. Clinical Pediatrics, 22, 241-244.

Siegel, L. S., \& Ryan, E. B. (1989). The development of working memory in normally achieving and subtypes of learning disabled children. Child Development, 60, 973-980.

Spellacy, F., \& Peter, B. (1978). Dyscalculia and elements of the developmental Gerstmann syndrome in school children. Cortex, 14(2), 197-206.

Starkey, P., Spelke, E. S., \& Gelman, R. (1990). Numerical abstraction by human infants. Cognition, 36, 97-128.

Temple, C. (1991). Procedural dyscalculia and number fact dyscalculia: Double dissociation in developmental dyscalculia. Cognitive Neuropsychology, 8, 155-176.

Temple, C. M., \& Sherwood, S. (2002). Representation and retrieval of arithmetical facts: Developmental difficulties. Quarterly Journal of Experimental Psychology, 55A(3), 733-752.

Thioux, M., Seron, X., \& Pesenti, M. (1999). Functional neuroanatomy of the semantic system: The case for numerals. Brain and Language, 69(3), 488-490.

Wechsler, D. (1992). WISC III UK. Sidcup, Kent: The Psychological Corporation.

Woodcock, R. W., McGrew, K. S., \& Mather, N. (2001). Woodcock-Johnson III. Itasca, IL: Riverside Publishing.

Wynn, K. (1992). Addition and subtraction by human infants. Nature, 358, 749-751.

Wynn, K. (1995). The origins of numerical knowledge. Mathematical Cognition, 1, 35-60. 